\title{
On p-radial Blaschke and harmonic Blaschke additions
}

\section{Chang-Jian Zhao*}

\section{"Correspondence:} chjzhao@163.com

Department of Mathematics, China

Jiliang University, Hangzhou,

310018, P.R. China

\begin{abstract}
In the paper, we first improve the radial Blaschke and harmonic Blaschke additions and introduce the $p$-radial Blaschke and $p$-harmonic Blaschke additions. Following this, Dresher type inequalities for the radial Blaschke-Minkowski homomorphisms with respect to $p$-radial Blaschke and $p$-harmonic Blaschke additions are established.

MSC: 46E27; 52A20

Keywords: radial Blaschke addition; harmonic Blaschke addition; -radial Blaschke addition; p-harmonic Blaschke addition; radial Blaschke-Minkowski homomorphisms; Brunn-Minkowski inequality
\end{abstract}

\section{Notation and preliminaries}

The setting for this paper is an $n$-dimensional Euclidean space $\mathbb{R}^{n}$. We reserve the letter $u$ for unit vectors, and the letter $B$ is reserved for the unit ball centered at the origin. The surface of $B$ is $S^{n-1}$. The volume of the unit $n$-ball is denoted by $\omega_{n}$. We use $V(K)$ for the $n$-dimensional volume of a body $K$. Associated with a compact subset $K$ of $\mathbb{R}^{n}$, which is star-shaped with respect to the origin, is its radial function $\rho(K, \cdot): S^{n-1} \rightarrow \mathbb{R}$ defined for $u \in S^{n-1}$ by

$$
\rho(K, u)=\max \{\lambda \geq 0: \lambda u \in K\}
$$

If $\rho(K, \cdot)$ is positive and continuous, $K$ will be called a star body. Let $\mathcal{S}^{n}$ denote the set of star bodies in $\mathbb{R}^{n}$. Let $\tilde{\delta}$ denote the radial Hausdorff metric, i.e., if $K, L \in \mathcal{S}^{n}$, then $\tilde{\delta}(K, L)=|\rho(K, u)-\rho(L, u)|_{\infty}$, where $|\cdot|_{\infty}$ denotes the sup-norm on the space of continuous functions $C\left(S^{n-1}\right)$.

\subsection{Dual mixed volumes}

The radial Minkowski linear combination, $\lambda_{1} K_{1} \widetilde{+} \cdots \widetilde{+} \lambda_{r} K_{r}$ is defined by

$$
\lambda_{1} K_{1} \tilde{+} \cdots \widetilde{+} \lambda_{r} K_{r}=\left\{\lambda_{1} x_{1} \widetilde{+} \cdots \widetilde{+} \lambda_{r} x_{r}: x_{i} \in K_{i}, i=1, \ldots, r\right\}
$$

for $K_{1}, \ldots, K_{r} \in \mathcal{S}^{n}$ and $\lambda_{1}, \ldots, \lambda_{r} \in \mathbb{R}$. It has the following important property (see [1]):

$$
\rho(\lambda K \widetilde{+} \mu L, \cdot)=\lambda \rho(K, \cdot)+\mu \rho(L, \cdot)
$$

(c) The Author(s) 2017. This article is distributed under the terms of the Creative Commons Attribution 4.0 International License (http://creativecommons.org/licenses/by/4.0/), which permits unrestricted use, distribution, and reproduction in any medium, provided you give appropriate credit to the original author(s) and the source, provide a link to the Creative Commons license, and indicate if changes were made. 
for $K, L \in \mathcal{S}^{n}$ and $\lambda, \mu \geq 0$. For $K_{1}, \ldots, K_{r} \in \mathcal{S}^{n}$ and $\lambda_{1}, \ldots, \lambda_{r} \geq 0$, the volume of the radial Minkowski linear combination $\lambda_{1} K_{1} \widetilde{+} \cdots \widetilde{+} \lambda_{r} K_{r}$ is a homogeneous polynomial of degree $n$ in the $\lambda_{i}$,

$$
V\left(\lambda_{1} K_{1} \widetilde{+} \cdots \widetilde{+} \lambda_{r} K_{r}\right)=\sum_{i_{1}, \ldots, i_{n}=1}^{r} \tilde{V}\left(K_{i_{1}}, \ldots, K_{i_{n}}\right) \lambda_{i_{1}} \cdots \lambda_{i_{n}} .
$$

If we require the coefficients of the polynomial in (1.3) to be symmetric in their arguments, then they are uniquely determined. The coefficient $\widetilde{V}\left(K_{i_{1}}, \ldots, K_{i_{n}}\right)$ is nonnegative and depends only on the bodies $K_{i_{1}}, \ldots, K_{i_{n}}$. It is called the dual mixed volume of $K_{i_{1}}, \ldots, K_{i_{n}}$.

If $K_{1}, \ldots, K_{n} \in \mathcal{S}^{n}$, then the dual mixed volume $\widetilde{V}\left(K_{1}, \ldots, K_{n}\right)$ can be represented in the form (see [2])

$$
\widetilde{V}\left(K_{1}, \ldots, K_{n}\right)=\frac{1}{n} \int_{S^{n-1}} \rho\left(K_{1}, u\right) \cdots \rho\left(K_{n}, u\right) d S(u) .
$$

If $K_{1}=\cdots=K_{n-i}=K, K_{n-i+1}=\cdots=K_{n}=L$, then the dual mixed volume is written as $\widetilde{V}_{i}(K, L)$. If $L=B$, then the dual mixed volume $\widetilde{V}_{i}(K, L)=\widetilde{V}_{i}(K, B)$ is written as $\widetilde{W}_{i}(K)$. For $K, L \in \mathcal{S}^{n}$, the $i$ th dual mixed volume of $K$ and $L, \widetilde{V}_{i}(K, L)$ can be extended to all $i \in \mathbb{R}$ by

$$
\widetilde{V}_{i}(K, L)=\frac{1}{n} \int_{S^{n-1}} \rho(K, u)^{n-i} \rho(L, u)^{i} d S(u),
$$

where $i \in \mathbb{R}$. Thus, if $K \in \mathcal{S}^{n}$, then

$$
\widetilde{W}_{i}(K)=\frac{1}{n} \int_{S^{n-1}} \rho(K, u)^{n-i} d S(u) .
$$

\subsection{Mixed intersection bodies}

For $K \in \mathcal{S}^{n}$, there is a unique star body $\mathbf{I} K$ whose radial function satisfies, for $u \in S^{n-1}$,

$$
\rho(\mathbf{I} K, u)=v\left(K \cap E_{u}\right)
$$

where $v$ is $(n-1)$-dimensional dual volume. It is called the intersection body of $K$. The volume of the intersection body of $K$ is given by (see [1])

$$
V(\mathbf{I} K)=\frac{1}{n} \int_{S^{n-1}} v\left(K \cap E_{u}\right)^{n} d S(u) .
$$

The mixed intersection body of $K_{1}, \ldots, K_{n-1} \in \mathcal{S}^{n}$, denoted by $\mathbf{I}\left(K_{1}, \ldots, K_{n-1}\right)$, is defined by

$$
\rho\left(\mathbf{I}\left(K_{1}, \ldots, K_{n-1}\right), u\right)=\tilde{v}\left(K_{1} \cap E_{u}, \ldots, K_{n-1} \cap E_{u}\right),
$$

where $\tilde{v}$ is the $(n-1)$-dimensional dual mixed volume. If $K_{1}=\cdots=K_{n-i-1}=K, K_{n-i}=\cdots=$ $K_{n-1}=L$, then $\mathbf{I}\left(K_{1}, \ldots, K_{n-1}\right)$ is written as $\mathbf{I}_{i}(K, L)$. If $L=B$, then $\mathbf{I}_{i}(K, L)$ is written as $\mathbf{I}_{i} K$ and called the $i$ th intersection body of $K$. For $\mathbf{I}_{0} K$, we simply write $\mathbf{I} K$. 


\section{Improvement of the radial Blaschke addition}

Let us recall the concept of radial Blaschke addition defined by Lutwak [1]. Suppose that $K$ and $L$ are star bodies in $\mathbb{R}^{n}$, the radial Blaschke addition denoted by $K \widehat{+} L$ is a star body whose radial function is

$$
\rho(K \widehat{+} L, \cdot)^{n-1}=\rho(K, \cdot)^{n-1}+\rho(L, \cdot)^{n-1} .
$$

The dual Knesser-Süss inequality for the radial Blaschke addition was established by Lutwak [1]. If $K, L \in \mathcal{S}^{n}$, then

$$
V(K \widehat{+} L)^{(n-1) / n} \leq V(K)^{(n-1) / n}+V(L)^{(n-1) / n},
$$

with equality if and only if $K$ and $L$ are dilates.

In the section, we give a generalized concept of the radial Blaschke addition.

Definition 2.1 If $K, L \in \mathcal{S}^{n}, 0 \leq p<n-1$ and $\lambda, \mu>0$ (not both zero), the $p$-radial Blaschke linear combination of $K$ and $L$ denoted by $\lambda \diamond K \widehat{+}_{p} \mu \diamond L$ is a star body whose radial function is defined by

$$
\rho\left(\lambda \diamond K \widehat{+}_{p} \mu \diamond L, \cdot\right)^{n-p-1}=\lambda \rho(K, \cdot)^{n-p-1}+\mu \rho(L, \cdot)^{n-p-1} .
$$

From (2.3), it is easy to see that

$$
\lambda \diamond K=\lambda^{1 /(n-p-1)} K
$$

When $\lambda=\mu=1$, the $p$-radial Blaschke combination becomes the $p$-radial Blaschke addition $K \widehat{+}_{p} L$ and

$$
\rho\left(K \widehat{+}_{p} L, \cdot\right)^{n-p-1}=\rho(K, \cdot)^{n-p-1}+\rho(L, \cdot)^{n-p-1} .
$$

Obviously, when $p=0,(2.4)$ becomes (2.1).

In the following, we define the dual mixed quermassintegral with respect to the $p$-radial Blaschke addition. First, we show two propositions. The following proposition follows immediately from (2.3) with L'Hôpital's rule.

Proposition 2.2 Let $0 \leq p<n-1,0 \leq i<n$ and $\varepsilon>0$. If $K, L \in \mathcal{S}^{n}$, then

$$
\lim _{\varepsilon \rightarrow 0^{+}} \frac{\rho\left(K \widehat{+}_{p} \varepsilon \diamond L, u\right)^{n-i}-\rho(K, u)^{n-i}}{\varepsilon}=\frac{n-i}{n-p-1} \rho(K, u)^{p-i+1} \rho(L, u)^{n-p-1} .
$$

The following proposition follows immediately from Proposition 2.2 and (1.6).

Proposition 2.3 Let $0 \leq p<n-1,0 \leq i<n$ and $\varepsilon>0$. If $K, L \in \mathcal{S}^{n}$, then

$$
\begin{gathered}
\frac{n-p-1}{n-i} \lim _{\varepsilon \rightarrow 0^{+}} \frac{\widetilde{W}_{i}\left(K \widehat{+}_{p} \varepsilon \diamond L, u\right)-\widetilde{W}_{i}(K)}{\varepsilon} \\
=\frac{1}{n} \int_{S^{n-1}} \rho(K, u)^{p-i+1} \rho(L, u)^{n-p-1} d S(u) .
\end{gathered}
$$


Definition 2.4 For $0 \leq p<n-1,0 \leq i<n$ and $K, L \in \mathcal{S}^{n}$, the $p$-dual mixed quermassintegral of star bodies $K$ and $L$, denoted by $\widetilde{W}_{p, i}(K, L)$, is defined by

$$
\widetilde{W}_{p, i}(K, L)=\frac{1}{n} \int_{S^{n-1}} \rho(K, u)^{p-i+1} \rho(L, u)^{n-p-1} d S(u) .
$$

Obviously, when $K=L, \widetilde{W}_{p, i}(K, L)$ becomes the dual quermassintegral of star body $K$, i.e., $\widetilde{W}_{p, i}(K, K)=\widetilde{W}_{i}(K)$. Taking $i=0$ in $(2.7), \widetilde{W}_{p, i}(K, L)$ becomes the $p$-dual mixed volume $\widetilde{V}_{p}(K, L)$ and

$$
\widetilde{V}_{p}(K, L)=\frac{1}{n} \int_{S^{n-1}} \rho(K, u)^{p+1} \rho(L, u)^{n-p-1} d S(u) .
$$

From (2.7), combining Hölder's integral inequality (see [3]) gives the following.

Proposition 2.5 (Minkowski type inequality) If $K, L \in \mathcal{S}^{n}, 0 \leq i<n$ and $0 \leq p<n-1$, then

$$
\widetilde{W}_{p, i}(K, L)^{n-i} \leq \widetilde{W}_{i}(K)^{p-i+1} \widetilde{W}_{i}(L)^{n-p-1}
$$

with equality if and only if $K$ and $L$ are dilates.

Taking $i=0$ in (2.9), we have: If $K, L \in \mathcal{S}^{n}$ and $0 \leq p<n-1$, then

$$
\widetilde{V}_{p}(K, L)^{n} \leq V(K)^{p+1} V(L)^{n-p-1},
$$

with equality if and only if $K$ and $L$ are dilates. In the following, we establish the BrunnMinkowski inequality for the $p$-radial Blaschke addition.

Proposition 2.6 If $K, L \in \mathcal{S}^{n}, 0 \leq i<n$ and $0 \leq p<n-1$, then

$$
\widetilde{W}_{i}\left(K \widehat{+}_{p} L\right)^{(n-p-1) /(n-i)} \leq \widetilde{W}_{i}(K)^{(n-p-1) /(n-i)}+\widetilde{W}_{i}(L)^{(n-p-1) /(n-i)},
$$

with equality if and only if $K$ and $L$ are dilates.

Proof From (2.3) and (2.7), it is easily seen that the $p$-dual mixed quermassintegral $\widehat{W}_{p, i}(K, L)$ is linear with respect to the $p$-radial Blaschke addition and together with inequality (2.9) shows that

$$
\begin{aligned}
\widetilde{W}_{p, i}\left(Q, K \widehat{+}_{p} L\right) & =\widetilde{W}_{p, i}(Q, K)+\widetilde{W}_{p, i}(Q, L) \\
& \leq \widetilde{W}_{i}(Q)^{(p-i+1) /(n-i)}\left(\widetilde{W}_{i}(K)^{(n-p-1) /(n-i)}+\widetilde{W}_{i}(L)^{(n-p-1) /(n-i)}\right),
\end{aligned}
$$

with equality if and only if $K$ and $L$ are dilates of $Q$. Take $K \widehat{+}_{p} L$ for $Q$ in (2.12), recall that $\widetilde{W}_{p, i}(Q, Q)=\widetilde{W}_{i}(Q)$, inequality $(2.11)$ follows easy.

Taking $i=0$ in (2.11), we obtain that if $K, L \in \mathcal{S}^{n}$ and $0 \leq p<n-1$, then

$$
V\left(K \widehat{+}_{p} L\right)^{(n-p-1) / n} \leq V(K)^{(n-p-1) / n}+V(L)^{(n-p-1) / n},
$$


with equality if and only if $K$ and $L$ are dilates. Taking $p=0$ and $i=0$ in (2.11), (2.11) becomes the well-known dual Knesser-Süss inequality (2.2).

\section{Improvement of the harmonic Blaschke addition}

Let us recall the concept of harmonic Blaschke addition defined by Lutwak [4]. Suppose that $K$ and $L$ are star bodies in $\mathbb{R}^{n}$, the harmonic Blaschke addition denoted by $K \breve{+} L$ is defined by

$$
\frac{\rho(K \breve{+} L, \cdot)^{n+1}}{V(K \breve{+} L)}=\frac{\rho(K, \cdot)^{n+1}}{V(K)}+\frac{\rho(L, \cdot)^{n+1}}{V(L)} .
$$

Lutwak's Brunn-Minkowski inequality for the harmonic Blaschke addition was established (see [4]). If $K, L \in \mathcal{S}^{n}$, then

$$
V(K \breve{+} L)^{1 / n} \geq V(K)^{1 / n}+V(L)^{1 / n},
$$

with equality if and only if $K$ and $L$ are dilates.

In the section, we give an improved concept of the harmonic Blaschke addition.

Definition 3.1 For $0 \leq i<n, p<i-1$ and $K, L \in \mathcal{S}^{n}$, we define the $p$-harmonic Blaschke addition of star bodies $K$ and $L$ denoted by $K \breve{+}_{p} L$ and defined by

$$
\frac{\rho\left(K \breve{+}_{p} L, \cdot\right)^{n-p-1}}{\tilde{W}_{i}\left(K \breve{+}_{p} L\right)}=\frac{\rho(K, \cdot)^{n-p-1}}{\widetilde{W}_{i}(K)}+\frac{\rho(L, \cdot)^{n-p-1}}{\tilde{W}_{i}(L)} .
$$

The Brunn-Minkowski inequality for the $p$-harmonic Blaschke addition follows immediately from (1.6), (3.3) and Minkowski's integral inequality (see [3]).

Proposition 3.2 If $K, L \in \mathcal{S}^{n}, 0 \leq i<n$ and $p<i-1$, then

$$
\widetilde{W}_{i}\left(K \breve{+}_{p} L\right)^{-(p+1-i) /(n-i)} \leq \widetilde{W}_{i}(K)^{-(p+1-i) /(n-i)}+\widetilde{W}_{i}(L)^{-(p+1-i) /(n-i)},
$$

with equality if and only if $K$ and $L$ are dilates.

\section{Radial Blaschke-Minkowski homomorphisms}

Definition 4.1 ([5]) A map $\Psi: \mathcal{S}^{n} \rightarrow \mathcal{S}^{n}$ is called a radial Blaschke-Minkowski homomorphism if it satisfies the following conditions:

(a) $\Psi$ is continuous.

(b) For all $K, L \in \mathcal{S}^{n}$,

$$
\Psi(K \ddot{+} L)=\Psi(K) \widetilde{+} \Psi(L) .
$$

(c) For all $K, L \in \mathcal{S}^{n}$ and every $\vartheta \in S O(n)$,

$$
\Psi(\vartheta K)=\vartheta \Psi(K)
$$

where $S O(n)$ is the group of rotations in $n$ dimensions. 
Radial Blaschke-Minkowski homomorphisms are important examples of star body valued valuations. Their natural duals, Blaschke-Minkowski homomorphisms, are an important notion in the theory of convex body valued valuations (see, e.g., [6-12] and [1320]). In 2006, Schuster [5] established the following Brunn-Minkowski inequality for radial Blaschke-Minkowski homomorphisms of star bodies. If $K$ and $L$ are star bodies in $\mathbb{R}^{n}$, then

$$
V(\Psi(K \widetilde{+} L))^{1 / n(n-1)} \leq V(\Psi K)^{1 / n(n-1)}+V(\Psi L)^{1 / n(n-1)}
$$

with equality if and only if $K$ and $L$ are dilates.

If $K$ and $L$ are star bodies in $\mathbb{R}^{n}, p \neq 0$ and $\lambda, \mu \geq 0$, then $\lambda \cdot K \widetilde{+}_{p} \mu \cdot L$ is the star body whose radial function is given by (see, e.g., [21])

$$
\rho\left(\lambda \cdot K \widetilde{+}_{p} \mu \cdot L, \cdot\right)^{p}=\lambda \rho(K, \cdot)^{p}+\mu \rho(L, \cdot)^{p} .
$$

The addition ${\widetilde{\tau_{p}}}_{p}$ is called $L_{p}$-radial addition. The $L_{p}$ dual Brunn-Minkowski inequality states: If $K, L \in \mathcal{S}^{n}$ and $0<p \leq n$, then

$$
V\left(K \widetilde{+}_{p} L\right)^{p / n} \leq V(K)^{p / n}+V(L)^{p / n}
$$

with equality when $p \neq n$ if and only if $K$ and $L$ are dilates. The inequality is reversed when $p>n$ or $p<0$ (see [21]).

In 2013, an $L_{p}$ Brunn-Minkowski inequality for radial Blaschke-Minkowski homomorphisms was established in [22]: If $K$ and $L$ are star bodies in $\mathbb{R}^{n}$ and $0<p<n-1$, then

$$
V\left(\Psi\left(K \widetilde{+}_{p} L\right)\right)^{p / n(n-1)} \leq V(\Psi K)^{p / n(n-1)}+V(\Psi L)^{p / n(n-1)},
$$

with equality if and only if $K$ and $L$ are dilates. Taking $p=1,(4.3)$ reduces to (4.1).

Theorem 4.2 (see [5]) Let $\Psi: \mathcal{S}^{n} \rightarrow \mathcal{S}^{n}$ be a radial Blaschke-Minkowski homomorphism. There is a continuous operator $\Psi: \underbrace{\mathcal{S}^{n} \times \cdots \times \mathcal{S}^{n}}_{n-1} \rightarrow \mathcal{S}^{n}$ symmetric in its arguments such that, for $K_{1}, \ldots, K_{m} \in \mathcal{S}^{n}$ and $\lambda_{1}, \ldots, \lambda_{m} \geq 0$,

$$
\Psi\left(\lambda_{1} K_{1} \widetilde{+} \cdots \widetilde{+} \lambda_{m} K_{m}\right)=\sum_{i_{1}, \ldots, i_{n-1}} \lambda_{i_{1}} \cdots \lambda_{i_{n-1}} \Psi\left(K_{i_{1}}, \ldots, K_{i_{n-1}}\right) .
$$

Clearly, Theorem 4.2 generalizes the notion of radial Blaschke-Minkowski homomorphisms. We call $\Psi: \mathcal{S}^{n} \times \cdots \times \mathcal{S}^{n} \rightarrow \mathcal{S}^{n}$ a mixed radial Blaschke-Minkowski homomorphism induced by $\Psi$. Mixed radial Blaschke-Minkowski homomorphisms were first studied in more detail in $[23,24]$. If $K_{1}=\cdots=K_{n-i-1}=K, K_{n-i}=\cdots=K_{n-1}=L$, we write $\Psi_{i}(K, L)$ for $\Psi(\underbrace{K, \ldots, K}_{n-i-1}, \underbrace{L, \ldots, L}_{i})$. If $K_{1}=\cdots=K_{n-i-1}=K, K_{n-i}=\cdots=K_{n-1}=B$, we write $\Psi_{i} K$ for $\Psi(\underbrace{K, \ldots, K}_{n-i-1}, \underbrace{B, \ldots, B}_{i})$ and call $\Psi_{i} K$ the mixed Blaschke-Minkowski homomorphism of order $i$ of $K . \Psi_{0} K$ is written simply as $\Psi K$. 
Lemma 4.3 (see [5]) A map $\Psi: \mathcal{S}^{n} \rightarrow \mathcal{S}^{n}$ is a radial Blaschke-Minkowski homomorphism if and only if there is a measure $\mu \in \mathcal{M}_{+}\left(S^{n-1}, \hat{e}\right)$ such that

$$
\rho(\Psi K, \cdot)=\rho(K, \cdot)^{n-1} * \mu,
$$

where $\mathcal{M}_{+}\left(S^{n-1}, \hat{e}\right)$ denotes the set of nonnegative zonal measures on $S^{n-1}$.

For the mixed radial Blaschke-Minkowski homomorphism induced by $\Psi$, Schuster [5] proved that

$$
\rho\left(\Psi\left(K_{1}, \ldots, K_{n-1}\right), \cdot\right)=\rho\left(K_{1}, \cdot\right) \cdots \rho\left(K_{n-1}, \cdot\right) * \mu .
$$

Obviously, a special case is the following:

$$
\rho\left(\Psi_{i} K, \cdot\right)=\rho(K, \cdot)^{n-1-i} * \mu,
$$

where $i$ are integers. We now extend the integers $i$ to real numbers, define the BlaschkeMinkowski homomorphism of order $p$ of $K$.

Definition 4.4 Let $K \in \mathcal{S}^{n}$, the Blaschke-Minkowski homomorphism of order $p$ of $K$, denoted by $\Psi_{p} K$, is defined for all $p \in \mathbb{R}$ by

$$
\rho\left(\Psi_{p} K, \cdot\right)=\rho(K, \cdot)^{n-1-p} * \mu .
$$

This extended definition will be required to prove our main results.

\section{Inequalities for the radial Blaschke-Minkowski homomorphism}

Theorem 5.1 Let $K, L \in \mathcal{S}^{n}$. If $0 \leq p<n-1$ and $i \leq n-1 \leq j \leq n$, then

$$
\left(\frac{\widetilde{W}_{i}\left(\Psi_{p}\left(K \widehat{+}_{p} L\right)\right)}{\widetilde{W}_{j}\left(\Psi_{p}\left(K \widehat{+}_{p} L\right)\right)}\right)^{1 /(j-i)} \leq\left(\frac{\widetilde{W}_{i}\left(\Psi_{p} K\right)}{\widetilde{W}_{j}\left(\Psi_{p} K\right)}\right)^{1 /(j-i)}+\left(\frac{\widetilde{W}_{i}\left(\Psi_{p} L\right)}{\widetilde{W}_{j}\left(\Psi_{p} L\right)}\right)^{1 /(j-i)},
$$

with equality if and only if $\Psi_{p} K$ and $\Psi_{p} L$ are dilates.

Remark 5.2 Taking $j=n$ in (5.1) and noting that $\widetilde{W}_{n}(K)=\int_{S^{n-1}} d S(u)=n \omega_{n}$, (5.1) becomes the following inequality: If $K, L \in \mathcal{S}^{n}, 0 \leq p<n-1$ and $i \leq n-1$, then

$$
\widetilde{W}_{i}\left(\Psi_{p}\left(K \widehat{+}_{p} L\right)\right)^{1 /(n-i)} \leq \widetilde{W}_{i}\left(\Psi_{p} K\right)^{1 /(n-i)}+\widetilde{W}_{i}\left(\Psi_{p} L\right)^{1 /(n-i)},
$$

with equality if and only if $\Psi_{p} K$ and $\Psi_{p} L$ are dilates. Taking $p=0$ in (5.1), (5.1) becomes the following inequality: If $K, L \in \mathcal{S}^{n}$ and $i \leq n-1 \leq j \leq n$, then

$$
\left(\frac{\widetilde{W}_{i}(\Psi(K \widehat{+} L))}{\widetilde{W}_{j}(\Psi(K \widehat{+} L))}\right)^{1 /(j-i)} \leq\left(\frac{\widetilde{W}_{i}(\Psi K)}{\widetilde{W}_{j}(\Psi K)}\right)^{1 /(j-i)}+\left(\frac{\widetilde{W}_{i}(\Psi L)}{\widetilde{W}_{j}(\Psi L)}\right)^{1 /(j-i)},
$$

with equality if and only if $\Psi K$ and $\Psi L$ are dilates. 
Theorem 5.3 Let $K, L \in \mathcal{S}^{n}$. If $0 \leq i<n, p<i-1$ and $k, j \in \mathbb{R}$ satisfy $j \leq n-1 \leq k \leq n$, then

$$
\begin{aligned}
& \frac{1}{\widetilde{W}_{i}\left(K \breve{+}_{p} L\right)}\left(\frac{\widetilde{W}_{j}\left(\Psi_{p}\left(K \breve{+}_{p} L\right)\right)}{\widetilde{W}_{k}\left(\Psi_{p}\left(K \breve{+}_{p} L\right)\right)}\right)^{1 /(k-j)} \\
& \leq \frac{1}{\widetilde{W}_{i}(K)}\left(\frac{\widetilde{W}_{j}\left(\Psi_{p} K\right)}{\widetilde{W}_{k}\left(\Psi_{p} K\right)}\right)^{1 /(k-j)}+\frac{1}{\widetilde{W}_{i}(L)}\left(\frac{\widetilde{W}_{j}\left(\Psi_{p} L\right)}{\widetilde{W}_{k}\left(\Psi_{p} L\right)}\right)^{1 /(k-j)},
\end{aligned}
$$

with equality if and only if $\Psi_{p} K$ and $\Psi_{p} L$ are dilates.

Remark 5.4 Taking $k=n$ in (5.4) and noting that $\widetilde{W}_{n}(K)=\int_{S^{n-1}} d S(u)=n \omega_{n}$, (5.4) becomes the following inequality: If $K, L \in \mathcal{S}^{n}, 0 \leq i<n, p<i-1$ and $j \leq n-1$, then

$$
\frac{\widetilde{W}_{j}\left(\Psi_{p}\left(K \breve{+}_{p} L\right)\right)^{1 /(n-j)}}{\widetilde{W}_{i}\left(K \breve{+}_{p} L\right)} \leq \frac{\widetilde{W}_{j}\left(\Psi_{p} K\right)^{1 /(n-j)}}{\widetilde{W}_{i}(K)}+\frac{\widetilde{W}_{j}\left(\Psi_{p} L\right)^{1 /(n-j)}}{\widetilde{W}_{i}(L)},
$$

with equality if and only if $\Psi_{p} K$ and $\Psi_{p} L$ are dilates. Taking $i=0, j=0$ and $k=n$ in (5.4), we have: If $K, L \in \mathcal{S}^{n}$ and $p<-1$, then

$$
\frac{V\left(\Psi_{p}\left(K \breve{+}_{p} L\right)\right)^{1 / n}}{V\left(K \breve{+}_{p} L\right)} \leq \frac{V\left(\Psi_{p} K\right)^{1 / n}}{V(K)}+\frac{V\left(\Psi_{p} L\right)^{1 / n}}{V(L)}
$$

with equality if and only if $\Psi_{p} K$ and $\Psi_{p} L$ are dilates.

\section{Dresher's inequalities for $\boldsymbol{p}$-radial Blaschke and harmonic Blaschke additions}

An extension of Beckenbach's inequality (see [3], p. 27) was obtained by Dresher [25] by means of moment-space techniques.

Lemma 6.1 (Dresher's inequality) If $p \geq 1 \geq r \geq 0, f, g \geq 0$ and $\phi$ is a distribution function, then

$$
\left(\frac{\int(f+g)^{p} d \phi}{\int(f+g)^{r} d \phi}\right)^{1 /(p-r)} \leq\left(\frac{\int f^{p} d \phi}{\int f^{r} d \phi}\right)^{1 /(p-r)}+\left(\frac{\int g^{p} d \phi}{\int g^{r} d \phi}\right)^{1 /(p-r)}
$$

with equality if and only if the functions $f$ and $g$ are proportional.

We are now in a position to prove Theorem 5.1. The following statement is just a slight reformulation of it.

Theorem 6.2 Let $K, L \in \mathcal{S}^{n}$. If $0 \leq p<n-1$ and $s, t \in \mathbb{R}$ satisfy $s \geq 1 \geq t \geq 0$, then

$$
\left(\frac{\widetilde{W}_{n-s}\left(\Psi_{p}\left(K \widehat{+}_{p} L\right)\right)}{\widetilde{W}_{n-t}\left(\Psi_{p}\left(K \widehat{+}_{p} L\right)\right)}\right)^{1 /(s-t)} \leq\left(\frac{\widetilde{W}_{n-s}\left(\Psi_{p} K\right)}{\widetilde{W}_{n-t}\left(\Psi_{p} K\right)}\right)^{1 /(s-t)}+\left(\frac{\widetilde{W}_{n-s}\left(\Psi_{p} L\right)}{\widetilde{W}_{n-t}\left(\Psi_{p} L\right)}\right)^{1 /(s-t)}
$$

with equality if and only if $\Psi_{p} K$ and $\Psi_{p} L$ are dilates.

Proof From (2.4), we obtain

$$
\rho\left(K \widehat{+}_{p} L, \cdot\right)^{n-p-1} * \mu=\rho(K, \cdot)^{n-p-1} * \mu+\rho(L, \cdot)^{n-p-1} * \mu,
$$


where $\mu$ is the generating measure of $\Psi$ from Lemma 4.3. Hence, from (4.6), we obtain

$$
\rho\left(\Psi_{p}\left(K \widehat{+}_{p} L\right), \cdot\right)=\rho\left(\Psi_{p} K, \cdot\right)+\rho\left(\Psi_{p} L, \cdot\right) .
$$

Therefore, by (1.6), we have

$$
\widetilde{W}_{n-s}\left(\Psi_{p}\left(K \widehat{+}_{p} L\right)\right)=\frac{1}{n} \int_{S^{n-1}}\left(\rho\left(\Psi_{p} K, u\right)+\rho\left(\Psi_{p} L, u\right)\right)^{s} d S(u)
$$

and

$$
\widetilde{W}_{n-t}\left(\Psi_{p}\left(K \widehat{+}_{p} L\right)\right)=\frac{1}{n} \int_{S^{n-1}}\left(\rho\left(\Psi_{p} K, u\right)+\rho\left(\Psi_{p} L, u\right)\right)^{t} d S(u)
$$

From (6.3), (6.4) and Lemma 6.1, we obtain

$$
\begin{aligned}
& \left(\frac{\widetilde{W}_{n-s}\left(\Psi_{p}\left(K \widehat{+}_{p} L\right)\right)}{\widetilde{W}_{n-t}\left(\Psi_{p}\left(K \widehat{+}_{p} L\right)\right)}\right)^{1 /(s-t)} \\
& \quad=\left(\frac{\int_{S^{n-1}}\left(\rho\left(\Psi_{p} K, u\right)+\rho\left(\Psi_{p} L, u\right)\right)^{s} d S(u)}{\int_{S^{n-1}}\left(\rho\left(\Psi_{p} K, u\right)+\rho\left(\Psi_{p} L, u\right)\right)^{t} d S(u)}\right)^{1 /(s-t)} \\
& \quad \leq\left(\frac{\int_{S^{n-1}} \rho\left(\Psi_{p} K, u\right)^{s} d S(u)}{\int_{S^{n-1}} \rho\left(\Psi_{p} K, u\right)^{t} d S(u)}\right)^{1 /(s-t)}+\left(\frac{\int_{S^{n-1}} \rho\left(\Psi_{p} L, u\right)^{s} d S(u)}{\int_{S^{n-1}} \rho\left(\Psi_{p} L, u\right)^{t} d S(u)}\right)^{1 /(s-t)} \\
& \quad=\left(\frac{\widetilde{W}_{n-s}\left(\Psi_{p} K\right)}{\widetilde{W}_{n-t}\left(\Psi_{p} K\right)}\right)^{1 /(s-t)}+\left(\frac{\widetilde{W}_{n-s}\left(\Psi_{p} L\right)}{\widetilde{W}_{n-t}\left(\Psi_{p} L\right)}\right)^{1 /(s-t)} .
\end{aligned}
$$

Equality holds if and only if the functions $\rho\left(\Psi_{p} K, u\right)$ and $\rho\left(\Psi_{p} L, u\right)$ are proportional.

Taking $s=n-i$ and $t=n-j$ in Theorem 6.2, Theorem 6.2 becomes Theorem 5.1 stated in Section 5. If $\Psi: \underbrace{\mathcal{S}^{n} \times \cdots \times \mathcal{S}^{n}}_{n-1} \rightarrow \mathcal{S}^{n}$ is the mixed intersection operator I : $\underbrace{\mathcal{S}^{n} \times \cdots \times \mathcal{S}^{n}}_{n-1} \rightarrow \mathcal{S}^{n}$ in (6.2) and $n-s=i$ and $n-t=j$, we obtain the following result: If $K, L \in \mathcal{S}^{n}, 0 \leq p<n-1$ and $i \leq n-1 \leq j \leq n$, then

$$
\left(\frac{\widetilde{W}_{i}\left(\mathbf{I}_{p}\left(K \widehat{+}_{p} L\right)\right)}{\widetilde{W}_{j}\left(\mathbf{I}_{p}\left(K \widehat{+}_{p} L\right)\right)}\right)^{1 /(j-i)} \leq\left(\frac{\widetilde{W}_{i}\left(\mathbf{I}_{p} K\right)}{\widetilde{W}_{j}\left(\mathbf{I}_{p} K\right)}\right)^{1 /(j-i)}+\left(\frac{\widetilde{W}_{i}\left(\mathbf{I}_{p} L\right)}{\widetilde{W}_{j}\left(\mathbf{I}_{p} L\right)}\right)^{1 /(j-i)}
$$

with equality if and only if $\mathbf{I}_{p} K$ and $\mathbf{I}_{p} L$ are dilates. Taking $j=n$ in (6.5) and noting that $\widetilde{W}_{n}(K)=\int_{S^{n-1}} d S(u)=n \omega_{n},(6.5)$ becomes the following inequality: If $K, L \in \mathcal{S}^{n}, 0 \leq p<$ $n-1$ and $i \leq n-1$, then

$$
\widetilde{W}_{i}\left(\mathbf{I}_{p}\left(K \widehat{+}_{p} L\right)\right)^{1 /(n-i)} \leq \widetilde{W}_{i}\left(\mathbf{I}_{p} K\right)^{1 /(n-i)}+\widetilde{W}_{i}\left(\mathbf{I}_{p} L\right)^{1 /(n-i)}
$$

with equality if and only if $\mathbf{I}_{p} K$ and $\mathbf{I}_{p} L$ are dilates.

We are now in a position to prove Theorem 5.3. The following statement is just a slight reformulation of it. 
Theorem 6.3 Let $K, L \in \mathcal{S}^{n}$. If $0 \leq i<n, p<i-1$ and $s, t \in \mathbb{R}$ satisfy $s \geq 1 \geq t \geq 0$, then

$$
\begin{aligned}
& \frac{1}{\widetilde{W}_{i}\left(K \breve{+}_{p} L\right)}\left(\frac{\widetilde{W}_{n-s}\left(\Psi_{p}\left(K \breve{+}_{p} L\right)\right)}{\widetilde{W}_{n-t}\left(\Psi_{p}\left(K \breve{+}_{p} L\right)\right)}\right)^{1 /(s-t)} \\
& \quad \leq \frac{1}{\widetilde{W}_{i}(K)}\left(\frac{\widetilde{W}_{n-s}\left(\Psi_{p} K\right)}{\widetilde{W}_{n-t}\left(\Psi_{p} K\right)}\right)^{1 /(s-t)}+\frac{1}{\widetilde{W}_{i}(L)}\left(\frac{\widetilde{W}_{n-s}\left(\Psi_{p} L\right)}{\widetilde{W}_{n-t}\left(\Psi_{p} L\right)}\right)^{1 /(s-t)},
\end{aligned}
$$

with equality if and only if $\Psi_{p} K$ and $\Psi_{p} L$ are dilates.

Proof From (3.3), we obtain

$$
\frac{\rho\left(K \breve{+}_{p} L, \cdot\right)^{n-p-1} * \mu}{\widetilde{W}_{i}\left(K \breve{+}_{p} L\right)}=\frac{\rho(K, \cdot)^{n-p-1} * \mu}{\widetilde{W}_{i}(K)}+\frac{\rho(L, \cdot)^{n-p-1} * \mu}{\widetilde{W}_{i}(L)} .
$$

Hence, from (4.6), we obtain

$$
\frac{\rho\left(\Psi_{p}\left(K \breve{+}_{p} L\right), \cdot\right)}{\widetilde{W}_{i}\left(K \breve{+}_{p} L\right)}=\frac{\rho\left(\Psi_{p} K, \cdot\right)}{\widetilde{W}_{i}(K)}+\frac{\rho\left(\Psi_{p} L, \cdot\right)}{\widetilde{W}_{i}(L)} .
$$

By (1.6), we have

$$
\frac{\widetilde{W}_{n-s}\left(\Psi_{p}\left(K \breve{+}_{p} L\right)\right)}{\widetilde{W}_{i}\left(K \breve{+}_{p} L\right)^{s}}=\frac{1}{n} \int_{S^{n-1}}\left(\frac{\rho\left(\Psi_{p} K, u\right)}{\widetilde{W}_{i}(K)}+\frac{\rho\left(\Psi_{p} L, u\right)}{\widetilde{W}_{i}(L)}\right)^{s} d S(u)
$$

and

$$
\frac{\widetilde{W}_{n-t}\left(\Psi_{p}\left(K \breve{+}_{p} L\right)\right)}{\widetilde{W}_{i}\left(K \breve{+}_{p} L\right)^{t}}=\frac{1}{n} \int_{S^{n-1}}\left(\frac{\rho\left(\Psi_{p} K, u\right)}{\widetilde{W}_{i}(K)}+\frac{\rho\left(\Psi_{p} L, u\right)}{\widetilde{W}_{i}(L)}\right)^{t} d S(u) .
$$

From (6.7), (6.8) and Lemma 6.1, we obtain

$$
\begin{aligned}
& \frac{1}{\widetilde{W}_{i}\left(K \breve{+}_{p} L\right)}\left(\frac{\widetilde{W}_{n-s}\left(\Psi_{p}\left(K \breve{+}_{p} L\right)\right)}{\widetilde{W}_{n-t}\left(\Psi_{p}\left(K \breve{+}_{p} L\right)\right)}\right)^{1 /(s-t)} \\
& =\left(\frac{\int_{S^{n-1}}\left(\frac{\rho\left(\Psi_{p} K, u\right)}{\widetilde{W}_{i}(K)}+\frac{\rho\left(\Psi_{p} L, u\right)}{\widetilde{W}_{i}(L)}\right)^{s} d S(u)}{\int_{S^{n-1}}\left(\frac{\rho\left(\Psi_{p} K, u\right)}{\widetilde{W}_{i}(K)}+\frac{\rho\left(\Psi_{p} L, u\right)}{\breve{W}_{i}(L)}\right)^{t} d S(u)}\right)^{1 /(s-t)} \\
& \leq\left(\frac{\int_{S^{n-1}}\left(\frac{\rho\left(\Psi_{p} K, u\right)}{\widetilde{W}_{i}(K)}\right)^{s} d S(u)}{\int_{S^{n-1}}\left(\frac{\rho\left(\Psi_{p} K, u\right)}{\widetilde{W}_{i}(K)}\right)^{t} d S(u)}\right)^{1 /(s-t)}+\left(\frac{\int_{S^{n-1}}\left(\frac{\rho\left(\Psi_{p} L, u\right)}{\widetilde{W}_{i}(L)}\right)^{s} d S(u)}{\int_{S^{n-1}}\left(\frac{\rho\left(\Psi_{p} L, u\right)}{\widetilde{W}_{i}(L)}\right)^{t} d S(u)}\right)^{1 /(s-t)} \\
& =\frac{1}{\widetilde{W}_{i}(K)}\left(\frac{\widetilde{W}_{n-s}\left(\Psi_{p} K\right)}{\widetilde{W}_{n-t}\left(\Psi_{p} K\right)}\right)^{1 /(s-t)}+\frac{1}{\widetilde{W}_{i}(L)}\left(\frac{\widetilde{W}_{n-s}\left(\Psi_{p} L\right)}{\widetilde{W}_{n-t}\left(\Psi_{p} L\right)}\right)^{1 /(s-t)},
\end{aligned}
$$

with equality if and only if $\Psi_{p} K$ and $\Psi_{p} L$ are dilates.

Taking $s=n-j$ and $t=n-k$ in Theorem 6.3, Theorem 6.3 becomes Theorem 5.3 stated in Section 5. If $\Psi: \underbrace{\mathcal{S}^{n} \times \cdots \times \mathcal{S}^{n}}_{n-1} \rightarrow \mathcal{S}^{n}$ is the mixed intersection operator I : $\underbrace{\mathcal{S}^{n} \times \cdots \times \mathcal{S}^{n}}_{n-1} \rightarrow \mathcal{S}^{n}$ in (6.6) and $j=n-s$ and $k=n-t$, we obtain the following result: 
If $K, L \in \mathcal{S}^{n}, 0 \leq i<n, p<i-1$ and $j \leq n-1 \leq k \leq n$, then

$$
\begin{aligned}
& \frac{1}{\widetilde{W}_{i}\left(K \breve{+}_{p} L\right)}\left(\frac{\widetilde{W}_{j}\left(\mathbf{I}_{p}\left(K \breve{+}{ }_{p} L\right)\right)}{\widetilde{W}_{k}\left(\mathbf{I}_{p}\left(K \breve{+}_{p} L\right)\right)}\right)^{1 /(k-j)} \\
& \quad \leq \frac{1}{\widetilde{W}_{i}(K)}\left(\frac{\widetilde{W}_{j}\left(\mathbf{I}_{p} K\right)}{\widetilde{W}_{k}\left(\mathbf{I}_{p} K\right)}\right)^{1 /(k-j)}+\frac{1}{\widetilde{W}_{i}(L)}\left(\frac{\widetilde{W}_{j}\left(\mathbf{I}_{p} L\right)}{\widetilde{W}_{k}\left(\mathbf{I}_{p} L\right)}\right)^{1 /(k-j)},
\end{aligned}
$$

with equality if and only if $\mathbf{I}_{p} K$ and $\mathbf{I}_{p} L$ are dilates. Taking $k=n$ in (6.9) and noting that $\widetilde{W}_{n}(K)=\int_{S^{n-1}} d S(u)=n \omega_{n},(6.9)$ becomes the following inequality: If $K, L \in \mathcal{S}^{n}, 0 \leq i<n$, $p<i-1$ and $j \leq n-1$, then

$$
\frac{\widetilde{W}_{j}\left(\mathbf{I}_{p}\left(K \breve{+}_{p} L\right)\right)^{1 /(n-j)}}{\widetilde{W}_{i}\left(K \breve{+}_{p} L\right)} \leq \frac{\widetilde{W}_{j}\left(\mathbf{I}_{p} K\right)^{1 /(n-j)}}{\widetilde{W}_{i}(K)}+\frac{\widetilde{W}_{j}\left(\mathbf{I}_{p} L\right)^{1 /(n-j)}}{\widetilde{W}_{i}(L)},
$$

with equality if and only if $\mathbf{I}_{p} K$ and $\mathbf{I}_{p} L$ are dilates.

\section{Conclusions}

In the present study, we first revised and improved the concepts of radial Blaschke addition and harmonic Blaschke addition in an $L_{p}$ space. Following this, we established Dresher's inequalities (Brunn-Minkowski type) for the radial Blaschke-Minkowski homomorphisms with respect to the $p$-radial addition and the $p$-harmonic Blaschke addition.

\section{Funding}

The author's research is supported by the Natural Science Foundation of China (11371334).

Competing interests

The author declares that he has no competing interests.

Authors' contributions

C-JZ provided the questions and gave the proof for the main results. He read and approved the manuscript.

\section{Publisher's Note}

Springer Nature remains neutral with regard to jurisdictional claims in published maps and institutional affiliations.

Received: 22 September 2017 Accepted: 4 December 2017 Published online: 16 December 2017

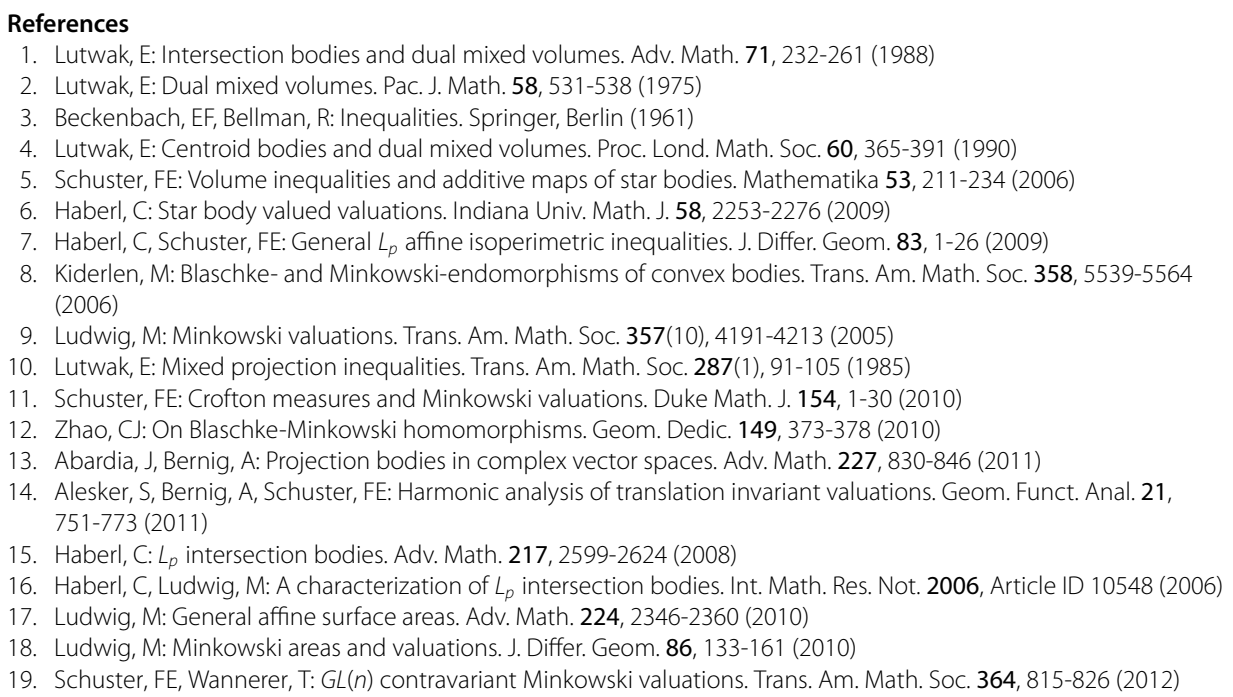


20. Wannerer, T: GL(n) equivariant Minkowski valuations. Indiana Univ. Math. J. 60, 1655-1672 (2011)

21. Gardner, RJ: The Brunn-Minkowski inequality. Bull. Am. Math. Soc. 39, 355-405 (2002)

22. Wang, W: $L_{p}$ Brunn-Minkowski type inequalities for Blaschke-Minkowski homomorphisms. Geom. Dedic. 164, 273-285 (2013)

23. Schuster, FE: Valuations and Busemann-Petty type problems. Adv. Math. 219, 344-368 (2008)

24. Schuster, FE: Convolutions and multiplier transformations of star bodies. Trans. Am. Math. Soc. 359, 5567-5591 (2007)

25. Dresher, M: Moment space and inequalities. Duke Math. J. 20, 261-271 (1953)

Submit your manuscript to a SpringerOpen ${ }^{\circ}$ journal and benefit from:

- Convenient online submission

- Rigorous peer review

- Open access: articles freely available online

- High visibility within the field

- Retaining the copyright to your article

Submit your next manuscript at $\boldsymbol{s p r i n g e r o p e n . c o m ~}$ 